\title{
Neuroendocrine tumors of the stomach: chemotherapy with cisplatin plus irinotecan is effective for gastric poorly-differentiated neuroendocrine carcinoma
}

\author{
Natsuko Tsuda Okita $\cdot$ Ken Kato $\cdot$ Daisuke Takahari $\cdot$ Yoshinori Hirashima \\ Takako E. Nakajima $\cdot$ Junichi Matsubara $\cdot$ Tetsuya Hamaguchi $\cdot$ \\ Yasuhide Yamada $\cdot$ Yasuhiro Shimada $\cdot$ Hirokazu Taniguchi $\cdot$ Kuniaki Shirao
}

Received: 5 August 2010/Accepted: 14 December 2010/Published online: 16 February 2011

(C) The International Gastric Cancer Association and The Japanese Gastric Cancer Association 2011

\begin{abstract}
Background Neuroendocrine tumors (NETs) occur in various primary sites, but rarely in the stomach. NETs are classified into three types, carcinoids, malignant carcinoids and poorly differentiated neuroendocrine carcinomas (PNECs), whose clinical behavior is different. Currently, clinical outcomes and standard chemotherapy for NETs of the stomach remain unclear.

Methods We conducted a retrospective review of histopathologically confirmed NETs of the stomach at our hospital between January 2000 and August 2006.

Results Thirty-seven NETs were identified. Fifteen patients had carcinoids while 22 had PNECs. Among the carcinoid patients, 7 underwent endoscopic mucosal resection and 5 had gastrectomy as first-line treatment. Three patients were observed without intervention. All patients were alive after an average follow-up period of 27 months. Among the 22 PNEC patients, 3 had no metastasis, 11 had regional lymph node metastasis, and 8 had distant metastasis. Eight of 14 patients relapsed at a median of 177 days (range 120-1459 days) after curative surgery. Twelve patients with metastatic or recurrent disease received palliative cisplatin plus irinotecan
\end{abstract}

N. T. Okita $(\bowtie) \cdot$ K. Kato - D. Takahari - Y. Hirashima ·

T. E. Nakajima - J. Matsubara - T. Hamaguchi - Y. Yamada .

Y. Shimada

Gastrointestinal Oncology Division, National Cancer Center

Hospital, 5-1-1 Tsukiji, Chuo-ku, Tokyo 104-0045, Japan

e-mail: natukot@gmail.com

H. Taniguchi

Clinical Laboratory Division, National Cancer Center Hospital,

Tokyo, Japan

K. Shirao

Clinical Oncology Division, Oita University, Oita, Japan chemotherapy. The response rate was $75 \%$, the median progression-free survival time was 212 days, and median survival time was 679 days.

Conclusion Gastric PNEC patients with distant metastasis had poor outcomes. Regimens containing cisplatin plus irinotecan produced a good response in gastric PNEC.

Keywords Carcinoid tumor - Poorly differentiated neuroendocrine carcinoma - Cisplatin · Irinotecan . Stomach

\section{Introduction}

Neuroendocrine tumors (NETs) occur in various primary sites, but rarely in the stomach. Gastric carcinoids account for only $3 \%$ of all carcinoid tumors [1]. The World Health Organization classifies endocrine tumors into the following categories: well-differentiated (neuro)endocrine tumors, well-differentiated (neuro)endocrine carcinomas, poorly differentiated (neuro)endocrine carcinomas (PNECs), and mixed exocrine-endocrine tumors [2]. Regarding NETs of the stomach, well-differentiated endocrine tumors and well-differentiated endocrine carcinomas are regarded as carcinoids and malignant carcinoids, respectively. PNEC is mainly regarded as small cell carcinoma.

Well-differentiated NETs and PNECs show different biological behaviors. Carcinoids demonstrate slow growth, whereas PNECs grow rapidly and carry a poor prognosis $[3,4]$. Carcinoids are treated by endoscopic mucosal resection (EMR) or surgery, and most patients with carcinoids have localized tumor and a good prognosis, with the 5-year survival for localized gastric carcinoids reported to be $93 \%$ [1]. Gastric PNECs without distant metastasis are mainly treated by surgical intervention, whereas PNECs 
with distant metastasis or recurrence are treated by chemotherapy. Various types of chemotherapy have been used to treat PNECs or extrapulmonary small cell carcinomas [4-7]; however, there is no standard regimen for metastatic or recurrent gastric PNECs.

Small cell lung cancer (SCLC) is a far more common disease than PNEC, but the two diseases share many clinicopathological features. In SCLC with extended disease, cisplatin plus irinotecan has been associated with better survival than cisplatin plus etoposide [8]. Here we report the clinical outcomes of primary NETs of the stomach and evaluate the efficacy of cisplatin plus irinotecan for gastric PNECs.

\section{Patients and methods}

This study was a retrospective analysis of histopathologically confirmed NETs primarily arising in the stomach in patients treated between January 2000 and August 2006 at the National Cancer Center Hospital in Tokyo, Japan. We extracted information on age, sex, disease stage, laboratory findings, radiological findings, pathological findings, therapy, effectiveness of therapy, and outcomes.

The cisplatin plus irinotecan regimen was administered as follows: on days 1 and 15 irinotecan $\left(70 \mathrm{mg} / \mathrm{m}^{2}\right)$ was given as a $90-\mathrm{min}$ intravenous infusion. Cisplatin $\left(80 \mathrm{mg} / \mathrm{m}^{2}\right)$ was given via 120 -min intravenous infusion on day 1 . This regimen was repeated every 4 weeks until the occurrence of tumor progression or severe adverse reactions. The chemotherapy response was evaluated according to the Response Evaluation Criteria in Solid Tumors (RECIST) guideline version 1.0. Toxicity was graded according to the Common Terminology Criteria for Adverse Events version 3.0.

Statistical analysis was performed using SPSS version 11 software (SPSS Japan., Tokyo, Japan). Overall survival and progression-free survival curves were constructed by the Kaplan-Meier method.

\section{Results}

Thirty-seven patients [male, $n=30$; female, $n=7$; median age, 67 years (range 27-82 years)] had NETs arising in the stomach. Of these 37 patients, 15 had carcinoids and 22 had PNECs (16 small cell carcinomas, 4 small cell carcinomas with adenocarcinoma, and 2 large cell carcinomas). Among the carcinoid patients, EMR was performed in 7 patients, including 3 who later underwent surgery; surgery was performed in 5 patients, and 3 patients were observed, due to complication (other cancer) or tumor disappearance after biopsy. No patient had metastasis or

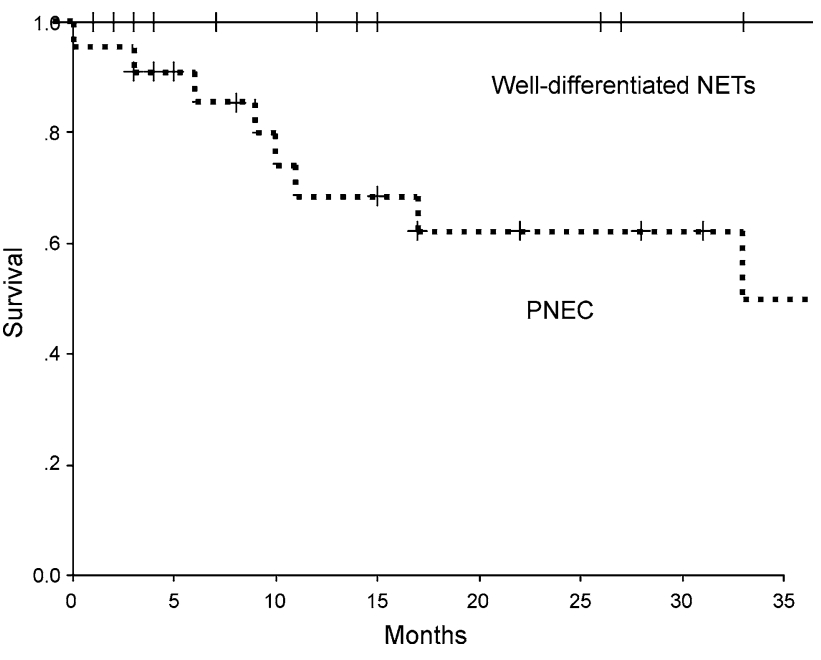

Fig. 1 Survival curves of patients with neuroendocrine tumors $(N E T s)$ primarily arising from the stomach. All patients with welldifferentiated type $(n=15)$ were alive, and the median survival time of patients with poorly differentiated neuroendocrine carcinoma (PNEC) was 33 months $(n=22)$

carcinoid syndrome. All the patients with carcinoids were alive after an average follow-up period of 27 months (Fig. 1).

The clinical data regarding PNEC patients are summarized in Table 1. Among the 22 PNEC patients, 14 had local disease and 8 had distant metastasis. Serum carcinoembryonic antigen (CEA), neuron-specific enolase (NSE), and pro-gastrin-releasing peptide (GRP) were elevated in $23 \%$ (5/22), 38\% (5/13), and 29\% (4/14) of patients, respectively. Surgery was performed in 15 patients (14 patients with localized disease and 1 patient in whom peritoneal dissemination was detected by cytologic diagnosis during surgery). Among the 14 patients with localized disease, 2 patients received adjuvant S-1 chemotherapy after curative surgery. Of those who underwent surgery, only 4 (27\%) were diagnosed with neuroendocrine carcinoma or small cell carcinoma from biopsy specimens prior to surgery.

Eight of 14 patients relapsed at a median of 177 days (range 120-1459 days) after curative surgery. A total of 14 patients ( 7 with unresectable disease at diagnosis, 7 with recurrent disease after surgery) received chemotherapy, and 1 relapsed patient received best supportive care.

The median survival time in the 22 PNEC patients was 33 months (Fig. 1), whereas in those with PNEC with distant metastasis the median survival time was 10.4 months. Twelve patients (5 with recurrent disease, 7 with unresectable disease) received chemotherapy with cisplatin plus irinotecan. The median number of treatment cycles was four. Six patients discontinued treatment because of disease progression, and 3 discontinued therapy because of adverse 
Table 1 Clinical data of patients with poorly differentiated neuroendocrine carcinomas

\begin{tabular}{|c|c|c|c|c|c|c|c|}
\hline Patient no. & Sex & Age (years) & Stage & $\mathrm{CEA}^{\mathrm{a}}$ & $\mathrm{NSE}^{\mathrm{b}}$ & $\mathrm{GRP}^{\mathrm{c}}$ & Treatment \\
\hline 1 & $\mathrm{M}$ & 61 & 1 & 3.7 & NE & NE & Surgery, RF \\
\hline 2 & M & 82 & 2 & 3.8 & $\mathrm{NE}$ & $\mathrm{NE}$ & Surgery, RC (liver), Palliation \\
\hline 3 & M & 71 & 2 & 1.1 & 8.5 & 13.9 & Surgery, RF \\
\hline 4 & $\mathrm{M}$ & 74 & 2 & 2.1 & 14 & 19.5 & Surgery, RC (liver), CX (IP) \\
\hline 5 & $\mathrm{M}$ & 72 & 2 & 2.5 & 33.6 & 44.6 & Surgery, RC (LN), CX (IP) \\
\hline 6 & M & 61 & 2 & 1.5 & 12.8 & 42.5 & Surgery, RC (liver), CX (IP) \\
\hline 7 & $\mathrm{~F}$ & 67 & 3 & 4 & NE & NE & Surgery, RF \\
\hline 8 & M & 75 & 3 & 2.7 & NE & NE & Surgery, RF \\
\hline 9 & $\mathrm{~F}$ & 70 & 3 & 8 & NE & $\mathrm{NE}$ & Surgery, RC (LN), CX (S-1) \\
\hline 10 & M & 59 & 3 & 2 & $\mathrm{NE}$ & $\mathrm{NE}$ & Surgery, AD (S-1), RF \\
\hline 11 & M & 69 & 3 & 6.7 & NE & $\mathrm{NE}$ & Surgery, RC (LN), CX (S-1) \\
\hline 12 & M & 60 & 3 & 51.6 & 12.7 & 1039 & Surgery, RC (liver), CX (IP) \\
\hline 13 & M & 75 & 3 & 4.5 & 9.7 & 21.2 & Surgery, AD (S-1), RF \\
\hline 14 & M & 62 & 3 & 3 & 8.4 & 21.2 & Surgery, RC (LN), CX (IP) \\
\hline 15 & $\mathrm{M}$ & 35 & 4 & 1.4 & 5.2 & 5.1 & Surgery, CX (IP) \\
\hline 16 & M & 68 & 4 & 1.4 & 197 & 18 & CX (IP) \\
\hline 17 & M & 62 & 4 & 7 & 89.6 & 5850 & CX (IP) \\
\hline 18 & M & 27 & 4 & 3.2 & NE & 138 & CX (IP) \\
\hline 19 & $\mathrm{~F}$ & 61 & 4 & 2 & 3.5 & 43.5 & CX (IP) \\
\hline 20 & M & 67 & 4 & 4.1 & 62.4 & 67.8 & CX (IP) \\
\hline 21 & M & 73 & 4 & 2.1 & 181.2 & 23.6 & CX (IP) \\
\hline 22 & M & 74 & 4 & 22.3 & $\mathrm{NE}$ & $\mathrm{NE}$ & Palliation \\
\hline
\end{tabular}

$N E$ not examined, $R F$ relapse-free, $R C$ recurrence (region), $C X$ chemotherapy, $I P$ irinotecan plus cisplatin, $A D$ adjuvant chemotherapy, $L N$ lymph node

${ }^{\text {a }}$ CEA carcinoembryonic antigen: normal range $<5 \mathrm{ng} / \mathrm{ml}$

${ }^{\mathrm{b}} N S E$ neuron-specific enolase: normal range $<15 \mathrm{ng} / \mathrm{ml}$

c $G R P$ pro-gastrin-releasing peptide: normal range $<46 \mathrm{pg} / \mathrm{ml}$

effects (neurotoxicity, febrile neutropenia, and diarrhea) at a median follow-up period of 29 months. The overall response rate was $75 \%(8 / 12)$ and 2 patients had stable disease. Rates of grade 3/4 neutropenia and diarrhea were $58 \%(7 / 12)$ and $17 \%$, respectively, but there were no treatment-related deaths. The median progression-free survival (PFS) time was 212 days (95\% confidence interval [CI] 121-302) and the median survival time was 679 days (95\% CI 39-1319). No patient had brain metastasis during the time of observation.

\section{Discussion}

NETs arise from the widely distributed neuroendocrine cell system. Histologically, NET cells are argentophilic and express endocrine markers such as chromogranin or NSE. NETs of the stomach are classified into the following three categories; carcinoids, malignant carcinoids, and PNECs or small cell carcinomas, on the basis of clinical and pathological features [2, 9]. However, some confusion remains with regard to the histological classification.

Gastric carcinoids account for about $0.3 \%$ of all gastric tumors. Gastrointestinal small cell carcinomas account for about $0.1-1.0 \%$ of all gastrointestinal tumors, and gastric small cell carcinomas account for approximately $11 \%$ of gastrointestinal small cell carcinomas [4]. As for NETs of the stomach, Rindi et al. [10] reviewed the histology of 55 gastric endocrine tumors and reported 46 cases of welldifferentiated tumors and 9 of neuroendocrine carcinomas. They further investigated 205 gastric endocrine tumors and reported 193 cases of well-differentiated and 12 of poorly differentiated tumors [11].

Well-differentiated NETs (carcinoids and malignant carcinoids) and PNECs have different biological behaviors. The 5-year survival rate of carcinoids without metastasis was reported to be 93-98\% [1, 3]. In carcinoids with metastasis, the 5-year survival rate was reported to be $0-75 \%$. The cumulative crude survival rate of PNEC was only $33.3 \%$ at 5 years after diagnosis. Carcinoid patients 
have better survival than patients with PNEC. In our study, the difference in survival between carcinoid and PNEC was similar to that in previous studies.

Standard treatment of patients with localized gastric carcinoids involves endoscopic resection or surgical excision [9]. For functioning tumors (carcinoid syndrome) as well, surgery is the primary treatment option. For patients with metastasis, management is not well defined and thus they are treated using several strategies, including surgery, biotherapy, and chemotherapy. Rinke et al. [12] reported that a somatostatin analog retarded tumor progression in patients with functionally active and inactive metastatic midgut NETs. We had only patients with small and/or localized carcinoids; thus, resection or observation was performed and they showed a good prognosis.

There are no standard chemotherapy regimens for gastric PNEC. Surgery is performed to treat localized disease in patients with gastric PNEC. Matsui et al. [13] reported 17 patients with gastric small cell carcinoma who underwent surgery; 3 patients without metastasis survived for 6-20 months after the surgery and 14 patients with metastasis died 5-22 months after the surgery at the end of their observation. In a review of 54 patients with gastric small cell carcinoma [14], 3 patients without distant metastasis survived for more than 2 years after gastrectomy with dissection of regional lymph nodes. In our study, although the relapse rate was high, there were 2 long-term survivors who showed no relapse for more than 3 years after surgery. Although the effectiveness of surgery is limited, it remains one of the most important modalities for treating gastric PNEC.

For patients with gastric adenocarcinoma, adjuvant S-1 chemotherapy is effective [15], and chemoradiotherapy is effective for limited stages of SCLC [16]. Further studies that explore factors associated with relapse and assess the efficacy of adjuvant chemotherapy or chemoradiotherapy for gastric PNEC are needed. Prophylactic cranial irradiation is standard therapy in patients with limited SCLC, due to the high rate of brain metastasis [17]. In our study, no patients had brain recurrence; thus, it is unclear whether or not prophylactic cranial irradiation is effective for gastric PNEC.

There is no standard regimen for metastatic or recurrent gastric PNECs, which are typically treated according to the treatment guidelines for SCLC. The standard chemotherapy for SCLC is a combination regimen containing cisplatin. Noda et al. [8] reported that patients treated with cisplatin plus irinotecan had better outcomes than patients treated with cisplatin plus etoposide, with median survival times in the two groups of 12.8 and 9.4 months, respectively. On the other hand, there are few reports on chemotherapy for PNEC other than the chemotherapy used for SCLC. Moertel et al. [5] reported that a regimen containing cisplatin plus etoposide produced a good response rate $(67 \%)$ in 18 patients with neuroendocrine carcinomas. Mitry et al. [6] obtained a response rate of $41.5 \%$ in 41 PNEC patients, with a PFS of 8.9 months and an overall survival of 15 months. These studies included only a few patients with gastric PNECs. A previous study showed good response of PNEC to combination chemotherapy with paclitaxel, carboplatin, and etoposide; however, the study included only 1 patient with gastric neuroendocrine carcinoma [7]. Kulke et al. [18] reported a very low response rate of $6.6 \%$ to cisplatin plus irinotecan for extrapulmonary NETs, although $78 \%(14 / 18)$ of their patients had welldifferentiated NETs.

In our study, treatment with cisplatin plus irinotecan was effective against gastric PNECs, with an overall response rate of $75 \%$ and a PFS of 212 days. We consider the toxicity of this regimen tolerable. Of note, gastric PNECs often have components of adenocarcinoma [13], and cisplatin plus irinotecan has been shown to be effective against gastric adenocarcinoma [19]. Therefore, we consider this regimen suitable for gastric PNEC, although the present retrospective study has several limitations. We are now planning a prospective study of this cisplatin plus irinotecan regimen in PNEC patients.

Acknowledgments We received no financial support for this work.

\section{References}

1. Godwin JD. Carcinoid tumors. Cancer. 1975;36:560-9.

2. Solcia E, Kloeppel G, Sobin LH. World Health Organization: International histological classification of tumours: histological typing of endocrine tumours. Berlin: Springer; 2000.

3. Botch K, Ahren B, Ahlman H, Falkmer S, Granerus G, Grimelius L. Gastric carcinoids. Biologic behavior and prognosis after differentiated treatment in relation to type. Ann Surg. 2005;242: 64-73.

4. Brenner B, Tang LH, Klimstra DS, Kelsen DP. Small-cell carcinomas of the gastrointestinal tract: a review. J Clin Oncol. 2004;22:2730-9.

5. Moertel CG, Kvols LK, O'Connell MJ, Rubin J. Treatment of neuroendocrine carcinomas with combined etoposide and cisplatin. Cancer. 1991;68:227-32.

6. Mitry E, Baudin E, Ducreux M, Sabourin JC, Rufle P, Aparicio T, et al. Treatment of poorly differentiated neuroendocrine tumours with etoposide and cisplatin. Br J Cancer. 1999;81:1351-5.

7. Hainsworth JD, Spigel DR, Litchy S, Greco FA. Phase II trial of paclitaxel, carboplatin and etoposide in advanced poorly differentiated neuroendocrine carcinoma: a Minnie Pearl Cancer Research Network study. J Clin Oncol. 2006;24:3548-54.

8. Noda K, Nishiwaki Y, Kawahara M, Negoro S, Sugiura T, Yokoyama A, et al. Irinotecan plus cisplatin compared with etoposide plus cisplatin for extensive small-cell lung cancer. N Engl J Med. 2002;346:85-91.

9. Arnold R. Endocrine tumours of the gastrointestinal tract. Introduction: definition, historical aspects, classification, staging, prognosis and therapeutic options. Best Pract Res Clin Gastroenterol. 2005;19:491-505. 
10. Rindi G, Luinetti O, Cornaggia M, Capella C, Solcia E. Three subtypes of gastric argyrophil carcinoid and the gastric neuroendocrine carcinoma: a clinicopathologic study. Gastroenterology. 1993;104:994-1006.

11. Rindi G, Bordi C, Rappel S, Rosa ST, Stolte M, Solcia E. Gastric carcinoids and neuroendocrine carcinomas; pathogenesis, pathology and behavior. World J Surg. 1996;20:168-72.

12. Rinke A, Muller HH, Brittinger CS, Klose KJ, Barth P, Wied M, et al. Placebo-controlled, double-blind, prospective, randomized study on the effect of octreotide LAR in the control of tumor growth in patients with metastatic neuroendocrine midgut tumors: a report from the PROMID study group. J Clin Oncol. 2009;27:4656-63.

13. Matsui K, Kitagawa M, Miwa A, Kuroda Y, Tsuji M. Small cell carcinoma of the stomach: a clinicopathologic study of 17 cases. Am J Gastroenterol. 1991;86:1167-75.

14. Arai K, Matsuda M. Gastric small-cell carcinoma in Japan: a case report and review of the literature. Am J Clin Oncol. 1998;21:458-61.
15. Sakuramoto S, Sasako M, Yamaguchi T, Kinoshita T, Fujii M, Nashimoto A, et al. Adjuvant chemotherapy for gastric cancer with S-1, an oral fluoropyrimidine. $N$ Engl J Med. 2007;357:1810-20.

16. Pignon JP, Arriagata R, Ihde DC, Johnson DH, Perry MC, Souhami RL, et al. A meta-analysis of thoracic radiotherapy for small-cell lung cancer. N Engl J Med. 1992;327:1618-24.

17. Auperin A, Arriagada R, Pignon JP, Le Péchoux C, Gregor A, Stephens RJ, et al. Prophylactic cranial irradiation for patients with small-cell lung cancer in complete remission. N Engl J Med. 1999;341:476-84.

18. Kulke MH, Wu B, Ryan D, Enzinger PC, Zhu AX, Clark JW, et al. A phase II trial of irinotecan and cisplatin in patients with metastatic neuroendocrine tumors. Dig Dis Sci. 2006;51:1033-8.

19. Boku N, Ohtsu A, Shimada Y, Shirao K, S Seki, Saito H, et al. Phase II study of a combination of irinotecan and cisplatin against metastatic gastric cancer. J Clin Oncol. 1999;17:319-23. 